\title{
Study of the Effects of Radiation at the CERN Gamma Irradiation Facility on the CMS Drift Tubes Muon Detector for the HL-LHC
}

\section{Bárbara Álvarez González on behalf of the CMS Muon Group}

Universidad de Oviedo, Instituto Universitario de Ciencias y Tecnologías Espaciales de Asturias (ICTEA) Oviedo, Spain

E-mail: balvarez@cern.ch

\begin{abstract}
The CMS drift tubes (DT) muon detector, built for withstanding the LHC expected integrated and instantaneous luminosities, will also be used in the High Luminosity LHC (HL-LHC) at a 5 times larger instantaneous luminosity and, consequently, much higher levels of radiation, reaching about 10 times the LHC integrated luminosity. Initial irradiation tests of a spare DT chamber at the CERN gamma irradiation facility $(\mathrm{GIF}++)$, at large $(\sim \mathrm{O}(100))$ acceleration factor, showed aging effects resulting in a degradation of the DT cell performance [1]; however, full CMS simulations have shown almost no impact in the muon reconstruction efficiency over the full barrel acceptance and for the full integrated luminosity. A second spare DT chamber was moved inside the GIF++ bunker in October 2017. The chamber was irradiated at lower acceleration factors, and only 2 out of the 12 layers of the chamber were switched at working voltage when the radioactive source is active, being the other layers in standby. In this way the other layers despite the irradation remain non-aged and are used as reference and as a precise and unbiased telescope of muon tracks for the efficiency computation of the aged layers of the chamber, when set at working voltage for measurements. An integrated dose equivalent to two times the expected integrated luminosity of the HL-LHC run has been absorbed by this second spare DT chamber and the final impact on the muon reconstruction efficiency is under study. Direct inspection of some extracted aged anode wires presented a resistive deposition of materials. Investigation on the outgassing of cell materials and of the gas components used at the GIF++ are underway. Strategies to mitigate the aging effects are also being developed. From the long irradiation measurements of the second spare DT chamber, the effects of radiation in the performance of the DTs expected during the HLLHC run are presented.
\end{abstract}




\section{Introduction and motivation}

The CMS drift tubes (DT) muon detector will also be used in the HL-LHC at an expected five times larger instantaneous luminosity and, consequently, much higher levels of radiation, reaching about ten times the LHC integrated luminosity. Irradiation campaigns have been performed in order to assess the capability of operating under such conditions and to investigate the aging induced by radiation.

Integrated Dose and Luminosity

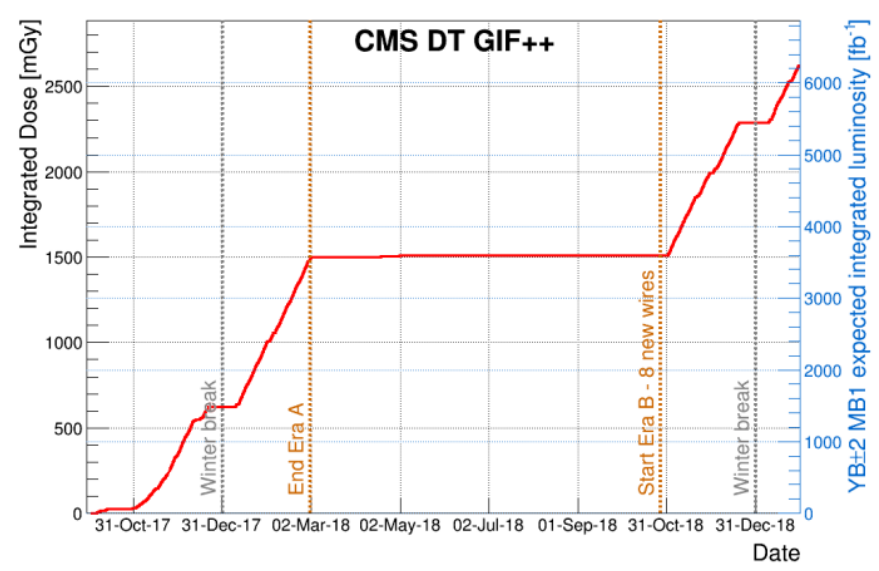

One DT chamber, MB2 DT spare chamber, installed at the CERN GIF++ facility [2] was irradiated with a high activity Cesium 137 source for a period of two years at different source intensities. An integrated dose equivalent to two times the expected integrated luminosity of the HL-LHC run has been absorbed by this spare DT chamber, Fig. 1, and the final impact on the muon reconstruction efficiency is under study. Strategies to mitigate the aging effects are also being developed.

Figure 1: Integrated dose [mGy] as a function of time for the irradiation period of the CMS DT spare chamber 'MB2' at GIF++ [3].

\section{Irradiation and data-taking}

The MB2 DT spare chamber was irradiated at a large $(\sim \mathrm{O}(10))$ acceleration factor, and only two out of the twelve layers of the chamber were switched on at working voltage $(3550 \mathrm{~V})$ when the radioactive source was active, being the other layers in standby $(1900 \mathrm{~V})$. The layers with high voltage off do not age when irradiated. In this way the other non-aged layers are used as reference and as a precise and unbiased telescope of muon tracks for the efficiency computation of the aged layers of the chamber.

Test beam muons and cosmic muons are used to measure the hit efficiency at the chamber under study. The hit efficiency for a DT cell in a layer is defined as the efficiency to detect a single hit in the given cell and it is measured as the ratio between the number of detected hits within the expected position from the track reconstructed with the other layers and the number of expected hits. It is important to highlight that the dose rate produced by the source and integrated dose absorbed by the chamber are converted to expected instantaneous and integrated luminosities at the HL-LHC from extrapolation of CMS Run2 measurements for the most exposed DT chambers located at high pseudorapidity in the innermost part of the muon system, the MB1 chambers on the external wheels $(\mathrm{YB} \pm 2)$.

\section{Results}

One of the main results of these studies is the evolution of the hit efficiency with respect to the integrated luminosity. Figure 2 shows the hit efficiency as a function of integrated luminosity for the aged layers (SL1L1 and SL1L4) at $3550 \mathrm{~V}$. Each of the points corresponds to the data-taking during HV scans (with the source off, no background rate) that were collected 
about every week. No atmospheric condition corrections have been applied. The hit efficiency is reduced less than $10 \%$ after integrating two times the HL-LHC expected luminosity.

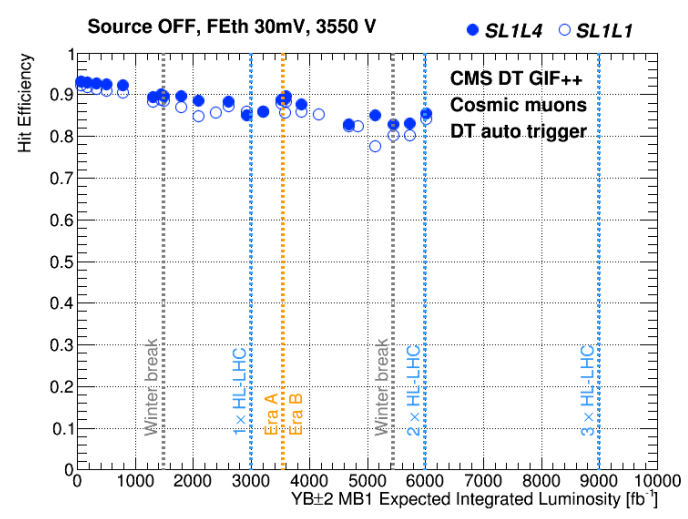

Figure 2: Hit efficiency for cosmic muons as a function of integrated luminosity for the aged layers (SL1L1 and SL1L4) at 3550 V [3].

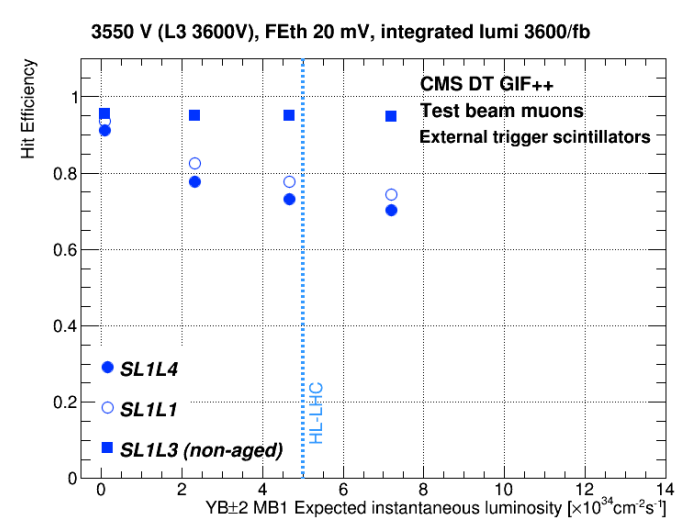

Figure 3: Hit efficiency for test beam muons as a function of instantaneous dose for the aged layers at $3550 \mathrm{~V}$ and for SL1L3 (non-aged) at $3600 \mathrm{~V}$ [3].

Another relevant result is shown in Figure 3 where the hit efficiency for test beam muons is presented as a function of instantaneous luminosity for the aged layers and for SL1L3 (nonaged) at a front-end threshold of $20 \mathrm{mV}$ after an irradiation integrated dose equivalent to a HLLHC integrated luminosity of $3600 \mathrm{fb}^{-1}$. A decrease of the hit efficiency of about $25 \%$ is observed at the HL-LHC background rate. Combining these results, the expected hit efficiencies at the end of the HL-LHC for all the DT chambers of the CMS muon system is estimated considering a safety factor of two for the expected HL-LHC background rate and a safety factor of two for the expected integrated luminosity. For the most exposed chambers, the MB1 chambers on the most external wheels, the hit efficiency drop is expected to be about $38 \%$ for these preliminary results. However, the expected efficiency drop of the rest of the DT system is much smaller due to the lower expected background rate.

\section{Conclusions}

HL-LHC will create a difficult environment for CMS subdetectors, and in particular for the Drift Tubes that may experience a degradation of performance for some chambers. A big effort has been made in order to fully characterize the radiation effects and to develop strategies to guarantee that the muon reconstruction and identification stay at an optimal level in CMS throughout the HL-LHC operation. Very preliminary studies including the expected hit efficiency at the end of the HL-LHC from GIF++ results implemented in the full CMS simulations have shown almost no impact on the muon reconstruction efficiency over the full barrel acceptance and for the full integrated luminosity. Further studies are ongoing in terms of trigger and rates.

\section{References}

[1] The Phase-2 Upgrade of the CMS Muon Detectors, , CMS Collaboration, CMS-TDR-016, https://cds.cern.ch/record/2283189

[2] CERN GIF++ M.R. Jaekel et al, 2014, PoS (TIPP2014) 102.

[3] Results of DT Longevity Studies, CMS Collaboration, CMS-DP-2019-018, https://cds.cern.ch/record/2682229 